\title{
Atypical skin reaction in a patient treated with gefitinib for advanced lung cancer: A case report and review of the literature
}

\author{
ANNA FERRAZZI $^{1}$, IRENE RUSSO ${ }^{1}$, GIULIA PASELLO ${ }^{2}$ and MAURO ALAIBAC ${ }^{1}$ \\ ${ }^{1}$ Dermatology Unit, Department of Medicine, University of Padua, Padua I-35121; \\ ${ }^{2}$ Unit of Medical Oncology, Veneto Institute of Oncology IOV-IRCCS, Padua I-35128, Italy
}

Received March 4, 2015; Accepted October 14, 2015

DOI: $10.3892 /$ etm.2015.2881

\begin{abstract}
Gefitinib is a selective epidermal growth factor receptor tyrosine kinase inhibitor utilized for the treatment of advanced non-small cell lung carcinoma. The most commonly reported adverse event during gefitinib therapy is skin rash, particularly a papulopustular acne-like eruption. Cutaneous toxicities can affect treatment compliance and the quality of life of the patient. The present study reports a case of gefitinib-induced atypical skin reaction in a 73-year-old woman with advanced non-small cell lung cancer, who developed a squamous-crusted eruption on her face after 4 weeks of oral treatment with gefitinib at a dose of $250 \mathrm{mg} /$ day. The patient was treated with $100 \mathrm{mg}$ minocyclin (2 tablets/day, orally) and with ryfamicin topically. A complete resolution of the lesions was observed 2 weeks later. The present case report explored the pathogenesis of this skin manifestation, focusing on the underlying immunological mechanisms. A review of the literature concerning skin reactions to gefitinib was also conducted.
\end{abstract}

\section{Introduction}

Epidermal growth factor receptor (EGFR) is a member of the of the EGFR family proteins, which consists of at least four receptor tyrosine kinases: EGFR, human epidermal growth factor receptor (HER)2, HER3 and HER4.

The EGFR family regulates the tyrosine kinase signaling pathway, which is responsible for cell cycle progression, division and differentiation. EGFR has been found to be overexpressed in certain types of solid tumors, such as colon, pancreas, lung and breast tumors, and leads to the dysregulation of cell growth (1).

Correspondence to: Professor Mauro Alaibac, Dermatology Unit, Department of Medicine, University of Padua, Via Battisti 206, Padua I-35121, Italy

E-mail: mauro.alaibac@unipd.it

Key words: gefitinib, epidermal growth factor receptor, skin immune system
Therapeutic agents with two main anti-EGFR mechanisms are used in clinical practice: Low molecular-weight tyrosine kinase inhibitors that compete with adenosine triphosphate (ATP) for binding with the tyrosine kinase portion of a mutant EGFR receptor, and monoclonal antibodies directed against the ligand-binding extracellular domain (1).

Agents targeting EGFR comprise some of the most extensively used drugs in the treatment of several common solid tumors (2). Gefitinib is an orally selective tyrosine kinase inhibitor of EGFR. Gefitinib and erlotinib were the first EGFR tyrosine kinase inhibitors to be approved by the US Food and Drug Administration for the treatment of advanced non-small cell lung carcinoma (NSCLC) (3).

Although these agents do not exhibit many of the classic side effects of cytotoxic chemotherapy, they are associated with unpleasant cutaneous toxicities, which can affect treatment compliance, as well as the quality of life of the patient $(2,4)$. Papulopustular acne-like eruption is the most common cutaneous adverse event (5) with an incidence rate of $55 \%$ (1). Xerosis, pruritus, desquamation, eczematous eruptions, teleangectasia, hyperpigmentation, trichomegalia, alopecia and nail alterations have also been reported (6-8). The present case report describes an atypical skin reaction in a patient treated with gefitinib.

\section{Case report}

The present study reports the case of a 73-year old woman suffering from advanced NSCLC, who developed a squamous, crusted eruption on her face after 4 weeks of treatment with gefitinib. The patient had been initially diagnosed with advanced lung adenocarcinoma in March 2013 and treated with gefinib at the Unit of Medical Oncology of the Veneto Institute of Oncology (Padua, Italy). The patient had never smoked and her initial symptoms were a persistent dry cough and a dull low back pain, which was unresponsive to corticosteroid therapy. A computed tomography (CT) scan revealed the presence of a mass in the left inferior lobe of the lung (average diameter, $5 \mathrm{~cm}$ ), which was found by histopathological examination to be a primary adenocarcinoma of the lung. Further examinations with positron emission tomography/CT revealed metastases in locoregional lymph nodes, bones and 
pleura. Magnetic resonance imaging (MRI) of the lumbar spine demonstrated secondary neoplastic lesions in multiple locations: D12, L2, L4, S1, S2 and the sacral right wing. The brain CT scan was negative. According to the TNM classification and staging system (9), lung adenocarcinoma stage IV was diagnosed and the EGFR mutation status (in-frame deletion of E746-750 in exon 19) was determined. The presence of an EGFR mutation-positive neoplasm was thus identified, and oral treatment with gefitinib was initiated at a dose of $250 \mathrm{mg}$ once daily. After 4 weeks of treatment with gefitinib, the patient visited the Dermatology Unit (Department of Medicine, University of Padua, Padua, Italy) in May 2013, presenting with a squamous-crusted eruption on her face (Fig. 1). The patient exhibited yellowish exophytic squamous-crusted lesions with sharp edges and an erythematous base. She had not previously applied topical products to the lesions, reported sun exposure or contact with aeroallergens, taken any other medications, or complained of fever, nausea, diarrhoea, abdominal pain or fatigue. The patient reported a weight loss of $3 \mathrm{~kg}$ during the last month prior to her visit to the hospital.

Dermatoscopic examination did not reveal abnormalities in the lesions, thus excluding the diagnosis of squamous cell carcinoma or seborrheic keratosis. The cutaneous swab was positive for Staphylococcus aureus. Complete blood count and liver and kidney function tests were normal, serum carcinoembryonic antigen was elevated $(1,300 \mathrm{ng} / \mathrm{ml})$ and an enzyme-linked immunosorbent assay for autoantibodies against desmogleins 1 and 3 was negative.

Treatment with gefitinib was discontinued and a systemic and topical antibiotic therapy was administered instead. The patient was treated with $100 \mathrm{mg}$ minocyclin ( 2 tablets/day) orally, and with rifamycin topically (twice a day for 1 month).

Two weeks later, the patient returned to the Unit exhibiting an almost complete resolution of the lesions (Fig. 2), and therefore gefitinib treatment (250 mg once daily) was resumed. Despite the gefitinib treatment, the disease progressed, leading to the mortality of the patient 2 months after resuming the treatment. Written informed consent was obtained from the patient for the publication of this case report and any accompanying images.

\section{Discussion}

EGFR inhibitors are widely used in monotherapy, or in combination with chemotherapy and/or radiotherapy, for the treatment of advanced solid cancers, such as NSCLC, squamous cell carcinoma of the head and neck, and colorectal and pancreatic cancer (10).

Among EGFR inhibitors, gefitinib is generally preferred because of its greater tolerability (2), despite the adverse events that are commonly reported, such as diarrhea, fatigue, nausea, elevated transaminase and skin rash (11). The most common adverse effects associated with EGFR inhibitors involve the skin. Several skin complications have been described, with the most frequent being an inflammatory papulopustular rash occurring within the first 2-4 weeks of treatment (12). The papulopustular eruption is usually distributed in the seborrheic areas and the primary lesions are follicular papules and pustules. Comedones are rarely observed (13). Histopathologically, a T-cell infiltrate around the follicular
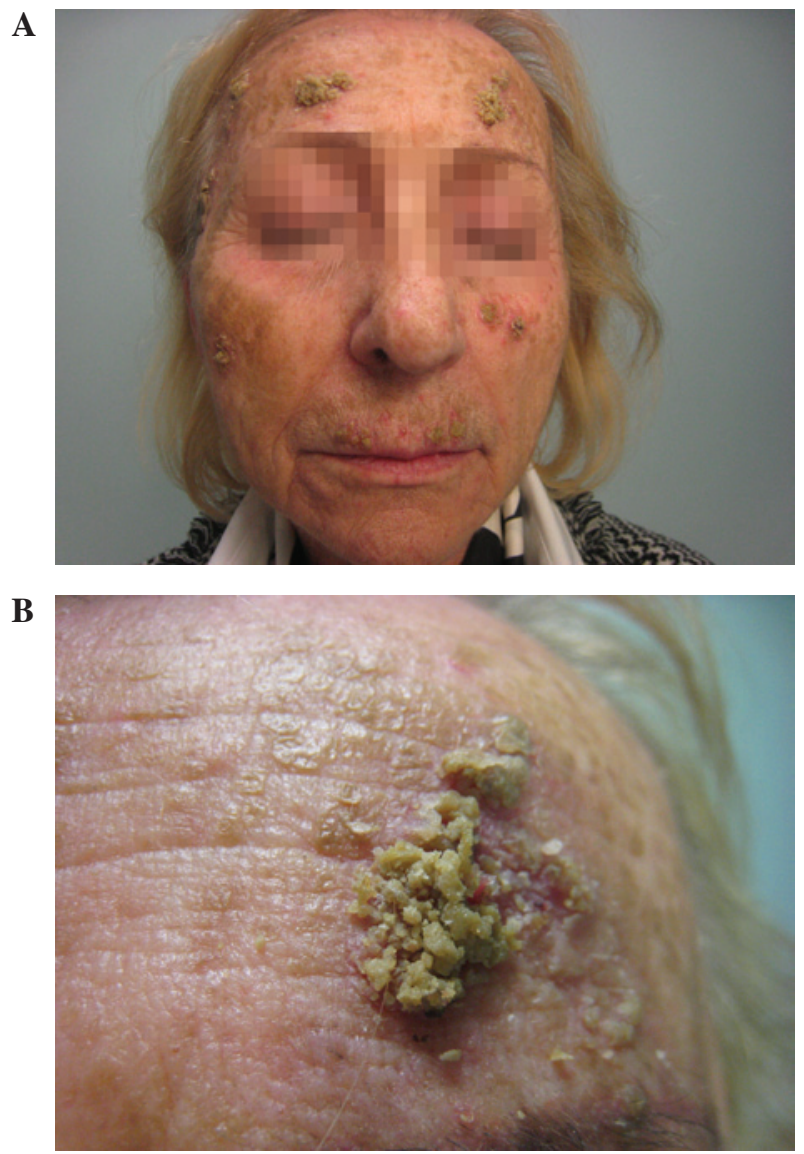

Figure 1. (A) Squamous-crusted eruption on the face of a 73-year old woman, who was undergoing oral treatment with gefitinib at an dose of $250 \mathrm{mg}$ once daily. (B) A yellowish exophytic squamous-crusted lesion with sharp edges and an erythematous base on the forehead of the patient.

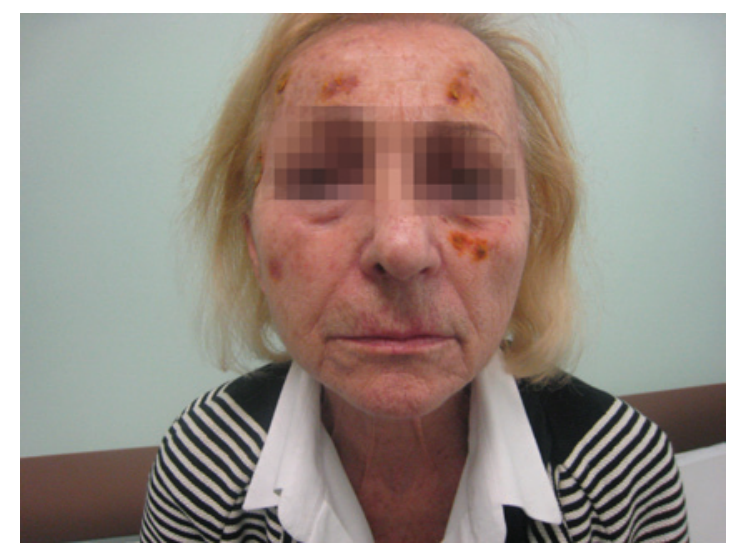

Figure 2. Almost complete resolution of the lesions on the face of the patient after 2 weeks of oral treatment with $100 \mathrm{mg}$ minocyclin ( 2 tablets/day) and topical treatment with rifamycin.

infundibulum is observed, which is associated with a suppurative folliculitis (14). Dry skin is very commonly observed in patients receiving treatment with EGFR inhibitors; vaginal dryness and itching, perineal dryness and blepharitis have been reported (15). The second most frequent systemic adverse effect of EGFR inhibitors is diarrhea, whose development can be associated with skin rash (16). Other cutaneous adverse reactions include paronychia, mucositis and hair 
changes, such as scalp alopecia, curling of the hair and facial hypertrichosis (17). Nail alterations are generally observed between weeks 4 and 8 from the initiation of gefitinib (13). The big toe is often affected by paronychia, which can be very painful if pyogenic granuloma of the nail fold develops (17). The combination of the papulopustular eruption, xerosis and nail and hair alterations along with pruritus is specific for this class of agents. The term PRIDE syndrome (papulopustules and/or paronychia, regulatory abnormalities of hair growth, itching, dryness caused by epidermal growth factor inhibitors) has been proposed for EGFR inhibitor-associated cutaneous complications $(18,19)$. Gefitinib-induced skin lesions usually occur on the face, scalp and upper chest and back, but can be observed anywhere (16). The skin rash, particularly when it occurs on the face, can affect the quality of life of the patient, and it may even result in a modification or discontinuation of their treatment. Furthermore, a high prevalence of cutaneous bacterial infections has been reported among patients with dermatological toxic effects following treatment with EGFR inhibitors (20). Other reported skin complications associated with gefitinib treatment are small-vessel vasculitis (21), psoriasis (22) and necrolytic migratory erythema (23).

To the best of our knowledge, the present study reports the first case of a squamous-crusted eruption on the face of a patient undergoing treatment with gefitinib. The epidermal alterations that this patient exhibited could have been the result of the activation of both innate and acquired immunity (16), leading to the production of cytokines acting on keratinocyte proliferation. A role of EGFR in the control of skin inflammation has been proposed on the basis of the observation that mice with an epidermis-restricted dominant negative EGFR mutation display an abundant inflammatory infiltrate in their skin, formed by macrophages, lymphocytes and granulocytes, starting 4-6 days after birth and progressively aggravating with the passage of time (24). Mascia et al (25) demonstrated that pharmacological blockade of EGFR boosted the expression of monocytes, dendritic cells and the T-cell chemoattractants chemokine (C-C motif) ligand (CCL) 2 and CCL5, as well as the T-cell-selective chemokine (C-X-C motif) ligand (CXCL)10, while it markedly impaired the expression of granulocytemacrophage colony-stimulating factor and CXCL8. These molecular events are dependent upon the concentration of the EGFR inhibitor, documenting the existence of an EGFR-driven regulatory mechanism during the keratinocyte response to inflammatory triggers (16). A strong upregulation of CCL2 and CCL5, but also of CCL27 and CXCL14, was found in the lesional skin of patients with cancer, who were undergoing treatment with EGFR inhibitors $(26,27)$. Finally, upregulated transcripts of tumor necrosis factor- $\alpha$ and interleukin-1 $\beta$, as well as CCL2, CCL5, CCL11 and CCL22, were detected in the skin and circulation of mouse models with EGFR ablation in the epidermis during their first week of life, prior to infiltration by immune cell populations $(27,28)$.

Due to the lack of clarity concerning the etiology of the skin rash, there are no evidence-based recommendations for treating it. Prophylactic treatments with local or systemic antibiotics, such as minocycline, have been recommended $(13,29)$, and it is plausible that the effect of minocycline on gefitinib-induced skin complications is mediated by its anti-inflammatory activities (30).
Further studies are required in order to explain the mechanisms through which EGFR-targeting agents, such as gefitinib, induce cutaneous reactions. Additional studies may facilitate a more natural approach to the treatment of these dermatologic adverse effects and provide more information concerning the physiological role of EGFR in the skin immune system. Informing the patients about the nature of these unpleasant skin side effects may restore their faith in the use of EGFR-targeted cancer treatments and help to ensure compliance.

\section{References}

1. Wnorowski MA, de Souza A, Chachoua A and Cohen DE: The management of EGFR inhibitor adverse events: A case series and treatment paradigm. Int J Dermatol 51: 223-232, 2012.

2. Ocvirk J, Heeger S, McCloud P and Hofheinz RD: A review of the treatment options for skin rash induced by EGFR targeted therapies: Evidence from randomized clinical trials and meta-analysis. Radiol Oncol 47: 166-175, 2013.

3. Luo M and Fu LW: Redundunt kinase activation and resistance of EGFR-tyrosine kinase inhibitors. Am J Cancer Res 4: 608-628, 2014.

4. Ricciardi S, Tomao S and de Marinis F: Toxicity of targeted therapy in non-small cell lung cancer management. Clin Lung Cancer 10: 28-35, 2009.

5. Peréz-Soler R, Delord JP, Halpern A, Kelly K, Krueger J, Sureda BM, von Pawel J, Temel J, Siena S, Soulières D, et al: HER1/EGFR inhibitor-associated rash: Future directions for management and investigation outcomes from the HER1/EGFR inhibitor rash management forum. Oncologist 10: 345-356, 2005

6. Chen AP, Setser A, Anadkat MJ, Cotliar J, Olsen EA, Garden BC and Lacouture ME: Grading dermatologic adverse events of cancer treatments: The common terminology criteria for adverse events version 4.0. J Am Acad Dermatol 67: 1025-1039, 2012.

7. Balagula Y, Rosen ST and Lacouture ME: The emergence of supportive oncodermatology: The study of dermatologic adverse events to cancer therapies. J Am Acad Dermatol 65: 624-635, 2011.

8. Eames T, Grabein B, Kroth J and Wololenberg A: Microbiological analysis of epidermal growth factor receptor inhibitor therapy-associated paronychia. J Eur Acad Dermatol Venereol 24: 958-960, 2010.

9. Rami-Porta R, Crowley JJ and Goldstraw P: The revised TNM staging system for lung cancer. Ann Thorac Cardiovasc Surg 15: 4-9, 2009.

10. Ciardello F and Tortora G: EGFR antagonists in cancer treatment. N Engl J Med 358: 1160-1174, 2008.

11. Liu HB, Wu Y,LV TF, Yao YW, Xiao YY, Yuan DM and Song Y: Skin rash could predict the response to EGFR tyrosine kinase inhibitor and the prognosis for patients with non-small cell lung cancer: A systematic review and meta-analysis. PloS One 8: e55128, 2013.

12. Potthoff K, Hofheinz R, Hassel JC, Volkenandt M, Lordick F, Hartmann JT, Karthaus M, Riess H, Lipp HP, Hauschild A, et al: Interdisciplinary management of EGFR-inhibitor-induced skin reactions: A German expert opinion. Ann Oncol 22: 524-535, 2011.

13. Heidary N, Naik H and Burgin S: Chemotherapeutic agents and the skin: An update. J Am Acad Dermatol 58: 545-570, 2008

14. Herbst RS, LoRusso PM, Purdom M and Ward D: Dermatologic side effects associated with gefitinib therapy: Clinical experience and management. Clin Lung Cancer 4: 366-369, 2003.

15. Herbst RS, Maddox AM, Rothenberg ML, Small EJ, Rubin EH, Baselga J, Rojo F, Hong WK, Swaisland H, Averbuch SD, et al: Selective oral epidermal growth factor receptor tyrosine kinase inhibitor ZD1839 is generally well-tolerated and has activity in non-small-cell lung cancer and other solid tumors: Results of a phase I trial. J Clin Oncol 20: 3815-3825, 2002.

16. Pastore S, Lulli D and Girolomoni G: Epidermal growth factor receptor signalling in keratinocyte biology: Implications for skin toxicity of tyrosine kinase inhibitors. Arch Toxicol 88: 1189-1203, 2014.

17. Chang GC, Yang TY, Chen KC, Yin MC, Wang RC and Lin YC: Complications of therapy in cancer patients: Case 1. Paronychia and skin hyperpigmentation induced by gefitinib in advanced non-small-cell lung cancer. J Clin Oncol 22: 4646-4648, 2004. 
18. Lacouture ME and Lai SE: The PRIDE (Papulopustules and/or paronychia, Regulatory abnormalities of hair growth, Itching, and dryness due to epidermal growth factor receptor inhibitors) syndrome. Br J Dermatol 155: 852-854, 2006.

19. Madke B, Gole P, Kumar P and Khopkar U: Dermatological side effects of epidermal growth factor receptor inhibitors: 'PRIDE' complex. Indian J Dermatol 59: 271-274, 2014.

20. Eilers RE Jr, Gandhi M, Patel JD, Mulcahy MF, Agulnik M, Hensing T and Lacouture ME: Dermatologic infections in cancer patients treated with epidermal growth factor receptor inhibitor therapy. J Natl Cancer Inst 102: 47-53, 2010.

21. Kurokawa I, Endo K and Hirabayashi M: Purpuric drug eruption possibly due to gefinitib (Iressa). Int J Dermatol 44: 167-168, 2005.

22. Zorzou MP, Stratigos A, Efstathiou E and Bamias A: Exacerbation of psoriasis after treatment with an EGFR tyrosine kinase inhibitor. Acta Derm Venereol 84: 308-309, 2004.

23. Trojan A, Jacky E, Follath F and Dummer R: Necrolytic migratory erythema (glucagenoma)-like skin lesions induced by EGF-receptor inhibition. Swiss Med Wkly 133: 22-23, 2003.

24. Murillas R, Larcher F, Conti CJ, Santos M, Ullrich A and Jorcano JL: Expression of a dominant negative mutant of epidermal growth factor receptor in the epidermis of transgenic mice elicits striking alterations in hair follicle development and skin structure. EMBO J 14: 5216-5223, 1995.
25. Mascia F, Cataisson C, Lee TC, Threadgill D, Mariani V, Amerio P, Chandrasekhara C, Souto Adeva G, Girolomoni G, Yuspa SH and Pastore S: EGFR regulates the expression of granulocyte/macrophage colony-stimulating factor in vitro and in vivo. J Invest Dermatol 130: 682-693, 2010.

26. Yamaki M, Sugiura K, Muro Y, Shimoyama Y and Tomita Y: Epidermal growth factor receptor tyrosine kinase inhibitors induce CCL2 and CCL5 via reduction in IL-1R2 in keratinocytes. Exp Dermatol 19: 730-735, 2010.

27. Lichtenberger BM, Gerber PA, Holcmann M, Buhren BA, Amberg N, Smolle V, Schrumpf H, Boelke E, Ansari P, Mackenzie C, et al: Epidermal EGFR controls cutaneous host defense and prevents inflammation. Sci Transl Med 5: 199ra111, 2013.

28. Mascia F, Lam G, Keith C, Garber C, Steinberg SM, Kohn E and Yuspa SH: Genetic ablation of epidermal EGFR reveals the dynamic origin of adverse effects of anti-EGFR therapy. Sci Transl Med 5: 199ra110, 2013.

29. Scope A, Agero AL, Dusza SW, Myskowski PL, Lieb JA, Saltz L, Kemeny NE and Halpern AC: Randomized double-blind trial of prophylactic oral minocycline and topical tazarotene for cetuximab-associated acne-like eruption. J Clin Oncol 25: 5390-5396, 2007

30. Garrido-Mesa N, Zarzuelo A and Gálvez J: Mynocicline: Far beyond an antibiotic. Br J Pharmacol 169: 337-352, 2013. 